\title{
New Prospect for Optoelectronics
}

\author{
Sergei Pyshkin* \\ Institute of Applied Physics, Academy of Sciences of Moldova, Moldova
}

Received: December 21, 2015; Accepted: January 18, 2016; Published: January 20, 2016

*Corresponding author: Sergei Pyshkin, Institute of Applied Physics, Academy of Sciences of Moldova, Moldova, Tel: $+37322-7326$ 21; Fax: $+37322-73$ 81 49; E-mail: spyshkin@yahoo.com

\begin{abstract}
Author Note
This review article generalizes the phenomena in semiconductors which give a unique opportunity to radically improve the materials for electronic devices, proposing new efficient light emissive optoelectronic devices and fundamentals for solid state physics.

It is the author's hope that the data presented here will be interesting and useful for university professors teaching specific courses in solid state physics, semiconductors, optics, non-linear optics, laser application and materials science, as well as for their students. The author is interested in collaboration with relevant firms and institutions around the world applying the achieved results in commercial sphere in preparation of perfect - close to ideal - semiconductors and their nanoparticles for top quality electronic devices. The author would be glad to discuss the results with management of the various corporations in electronic industry and with various specialists, and to present a unique collection of long-term ordered GaP samples, prospectively establishing collaboration to develop the new methods of preparation of close to ideal materials.

The author is glad to note that the broad discussion and dissemination of our results stimulate successful collaboration with reliable partners from USA, Russia, Italy, Romania, France and other countries. The author is very grateful to the US Department of State, Fulbright Foundation, Institute of International Exchange, Washington, DC, The US Air Force Office for Scientific Research, the US Office of Naval Research Global, Civilian R\&D Foundation, Arlington, VA, to his colleagues and co-authors from Clemson University, SC, University of Central Florida, FL, Istituto di elettronica dello stato solido, CNR, Rome, Italy, Universita degli studi, Cagliari, Italy, Lomonosov Moscow State University, Ioffe Physical-Technical Institute of the Russian Academy of Sciences and Peter the Great St.Petersburg Polytechnic University, Prokhorov General Physics Institute, Russian Academy of Sciences, Moscow, Institute of Applied Physics and Academy of Sciences of Moldova for support and attention to this protracted (1961-present time) research.
\end{abstract}

\section{Abstract}

This article demonstrates the results of the development of technology for growth of perfect and free of contamination Gallium Phosphide (GaP) crystals, and the influence of crystallization conditions on quality and properties of the crystals. The long-term ordered and therefore close to ideal crystals repeat behavior of the best nanoparticles with pronounced quantum confinement effect. These perfect crystals are useful for application in top-quality optoelectronic devices as well as they are a new object in the development of fundamentals of solid state physics.

In the 1960s the first Gallium Phosphide crystals doped by nitrogen (GaP:N) were prepared by the author, followed by the introduction of the excitonic crystal concept in the 1970s. Since then, the author has developed arguably the best methods of bulk, film and nanoparticle GaP crystal growth. Novel and useful properties of perfect GaP, including its stimulated emission, very bright and broadband luminescence at room temperature, have been observed. This paper compiles the results of the semi-centennial evolution of GaP:N properties. These results provide the possibility of a new approach to selection and preparation of perfect materials for optoelectronics and a unique opportunity to realize a new form of solid-state host - the excitonic crystal as high intensity light source with expected low threshold for generation of non-linear optical effects.

Using the example of GaP, this article proposes a method for cheap, resource-saving and impactful way of the development of optoelectronics with the help of transformation of an ordinary semiconductor into base material for various device structures.

Keywords: GaP; Long-term ordering; Excitonic crystal

\section{Main Results and their Analysis}

Relevant research work started already in 1961. For more than 50 years, various investigations of GaP semiconductor were driven by the author individually or jointly with the wellknown scientists from Russia, USA, Italy and Moldova: Prof. John Ballato (Clemson University, SC, USA) and my teachers, the late professors N.A. Goryunova (Ioffe Institute, St. Petersburg, Russia), S.I. Radautsan (Academy of Sciences of Moldova, Kishinev), R.V. Khokhlov (Lomonosov Moscow State University), and Nobel Prize Laureate A.M. Prokhorov (Prokhorov General Physics Institute, Moscow, Russia). Main results, as they occurred and as they were understood and interpreted for more than half a century, are presented in many works. The most important ones are covered in [1-11]. Since pure and doped samples of Gallium Phosphide were originally prepared in the 1960s [1,2], followed by the introduction of the excitonic crystal concept in the 1970s [3], the author discovered stimulated emission with indirect optical transitions [4] and interesting non-linear optical 
effects [5] in this semiconductor. Novel and useful properties of the perfect $\mathrm{GaP}$ were observed, including its stimulated emission, very bright and broadband luminescence at room temperature. Arguably, the best methods of bulk, film and nanoparticle crystal growth have been elaborated since [6-11].

In particular, it is shown that, contrary to freshly-grown GaP crystals having no luminescence at $300 \mathrm{~K}$, perfect longterm ordered GaP crystals and their high quality nanoparticles between 1.2 and $3.2 \mathrm{eV}$ IR and UV emit bright luminescence, that is $1 \mathrm{eV}$ less or more than the nearly $2.3 \mathrm{eV} \mathrm{GaP}$ forbidden gap (Figures 1,2). It is important to note the unique possibility to alter the position of the luminescent maximum between IR and UV ranges by introducing certain dopants into the crystals and changing the level of excitation of the luminescence, or simply by choosing suitable thickness of the crystal.

For instance, the $10 \mathrm{~nm}$ GaP nanoparticles are nearly transparent for their UV luminescence with the maximum at $3.2 \mathrm{eV}$ (Figure 2, spectrum 3), while this $3.2 \mathrm{eV}$ emission will be completely absorbed inside the thick bulk GaP single crystals due to very high light absorption coefficient in this region (Figure 2, spectrum 1). Remembering that the ratio between initial $I_{0}$ and transmitted through any medium light $\mathrm{I}$ is $\mathrm{I}=\mathrm{I}_{0} \exp (-\mathrm{kx})$, where $\mathrm{k}$ and $\mathrm{x}$ are the absorption coefficient and the thickness of the absorbing layer, a negligible small contribution of absorption for GaP nanoparticles is observed despite the high absorption coefficient at $3.2 \mathrm{eV}$.

As it also shown in Figure 3b, [8], that the GaP:N crystals aged for at least 40 years possess no discrete impurity level for $\mathrm{N}$-bound excitons in the forbidden gap. Instead of the narrow zero-phonon line and its phonon replica observed from the lessaged 25-year-old crystals (Figure 3a) they also demonstrate a uniform luminescence from a broad excitonic band (Figure $3 b$ ). Additionally, stimulated emission of light in these temporally ordered crystals (Figure $3 \mathrm{~b}$ ) is observed.

Figures 1-3 demonstrate the differences between the disordered, newly prepared single crystals, and crystals ordered for 25 years, and for 40 years. As shown in Figure 1,3, differences

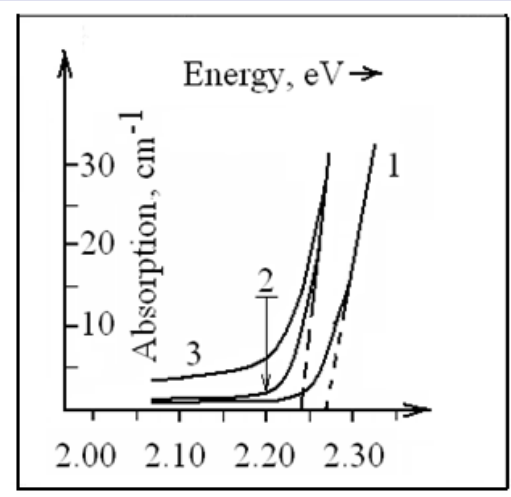

Figure 1: Forty-year evolution of absorption edge in GaP doped with different impurity concentration $\mathrm{N}_{0}\left(\mathrm{~cm}^{-3}\right)$. Absorption coefficient in long-term-ordered (40-year) GaP:N (1) and in 40-year-old doped (2) or freshly-prepared undoped GaP:N (3). N concentration is the same for lines 1 and 3.

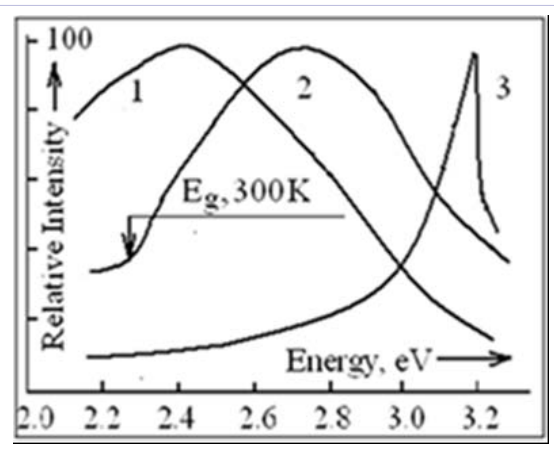

Figure 2: Luminescence of perfect GaP single crystals (1) in comparison with the luminescence of GaP nanoparticles, stored as the dry powder (2) or liquid suspension (3).

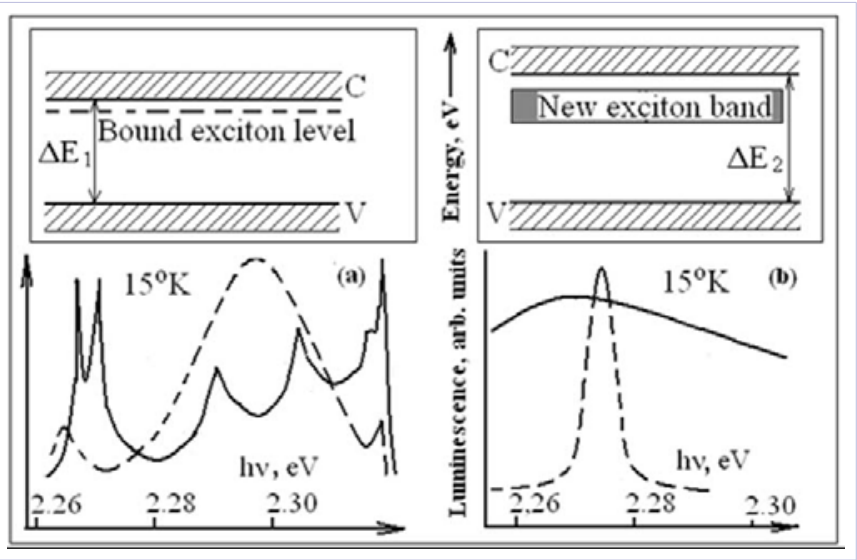

Figure 3: Luminescent spectra and schematic representation of the forbidden gaps $(\Delta \mathrm{E} 1, \Delta \mathrm{E} 2)$ in the nitrogen-doped GaP aged for (a) 25 years and (b) 40 years. 15-30K. The dotted lines correspond to highly optically excited crystals. $C$ and $V$ represent the positions of the bottom of the conductance and the top of valence bands, respectively.

between the freshly prepared and long-term ordered crystals are observed in the luminescent line-widths and their absorption edge spectral position with $\mathrm{N}$ concentration, suggesting an improvement with time in ordering of the host lattice and nearly equal spacing between impurities. The position of the absorption edge in freshly prepared GaP:N crystals does not depend on $\mathrm{N}$ impurity concentration. This impurity creates the 21-meV energy level under the conduction band for bound excitons both for freshly prepared and 25-year ordered crystals. On the contrary, the GaP:N crystals ordered for approximately 40 years demonstrate an increase in the forbidden gap, which is proportionate to the $\mathrm{N}$ concentration according to Vegard's law, similar to that in a dilute GaP-GaN solid solution. The 40year ordered GaP:N crystals have no discrete impurity level in the forbidden gap and demonstrate uniform luminescence from a broad excitonic band instead of narrow zero-phonon line and its phonon replica in disordered and partly ordered (25-year-old) crystals.

For the first time, to the best of our knowledge, the transformation of a discrete level inside the forbidden gap into an excitonic band can be observed (Figure 3b). In this case, 
the impurity atom regularly builds into host lattice and affects the band structure of the crystals, which is now a dilute solid solution of GaP-GaN with its own forbidden gap (Figure 1, curve 1) but not GaP crystals doped by occasionally located $\mathrm{N}$ atoms (Figure1, curve 2). Note, the increase of the level of excitation of luminescence (dotted lines in Figure 3) in the case of partly ordered GaP:N leads to a broad luminescence band as a result of bound exciton interaction. Meanwhile, in the case of perfectly ordered crystals, one can see an abrupt narrowing of the luminescence band, probably due to stimulated emission in perfect crystals.

While increasing the intensity of excitation, one can decrease the contribution into luminescence of light emissive recombination through donor (D)-acceptor (A) pairs, for instance $\mathrm{Ge}-\mathrm{O}$, when the concentration of these pairs will be equal and less than the concentration of electron-hole pairs generated by the laser excitation source. In these conditions, an increase with excitation of contribution into the total light emission from other recombination channels depressed by the recombination through Ge-O pairs will also be clearly detected. Thus, the infrared-visible light switching using various light emissive recombination channels, important for application in optoelectronic devices, is demonstrated in the luminescence of GaP doped by Ge. Figure 4 demonstrates, depending on excitation intensity, the switching of luminescence at $77.3 \mathrm{~K}$ - from infrared with maximum at 1.38 $\mathrm{eV}$ to green-yellow region with maximum at $2.06 \mathrm{eV} \mathrm{[10].} \mathrm{This}$ result shows a possibility to change the position of luminescent maximum choosing only a single semiconductor at suitable thickness of the crystal, impurities and the level of excitation. Thus, we can get a perfect, close to ideal, semiconductor light emission from IR to UV. Our results provide a new approach to selection and preparation of perfect materials for optoelectronics and a unique opportunity to develop a new form of solid-state host discussed below-the excitonic crystal as high intensity light source with expected low threshold for the generation of non-linear optical phenomena.

Bound excitons are the base for creation of the excitonic crystals. They are well studied in semiconductors, especially in Gallium Phosphide doped by nitrogen (GaP:N) [2,3,5]. Doping of GaP with $\mathrm{N}$ leads to isoelectronic substitution of the host $\mathrm{P}$ atoms by $\mathrm{N}$ in its crystal lattice and to the creation of the electron trap with a giant capture cross-section. Therefore, every non-equilibrium electron in the vicinity of the trap will be captured by $\mathrm{N}$ atom, attracting further a non-equilibrium hole by Coulomb interaction and creating the bound exciton-shortlived nanoparticle with the dimension of the order of $10 \mathrm{~nm}$ (it is the Bohr diameter of bound exciton in GaP:N). Note, that none of nanotechnology methods are used for creation or selection of the dimensions of these nanoparticles-only natural forces of electron-hole interaction and electron capture by the traps are necessary for the creation of these nanoparticles, important for application in various branches of electronic industry. As the result, we get a sort of neutral short-lived atom analogue-a particle consisting of heavy negatively charged nucleus ( $\mathrm{N}$ atom with captured electron) and a hole. So-called zero vibrations in the presence of these heavy nuclei do not destroy the possible solid phase of bound excitons. This gives an opportunity to reach a new crystal state in GaP:N - the short-lived excitonic crystal appears at a certain necessary level of excitation, $\mathrm{N}$ impurity ordering and concentration, energy of exciting photons and temperature.

A model for the GaP:N long-term ordered crystal and its behavior at the relevant level of optical excitation for 40-yearold ordered N-doped GaP is presented in Figure 5. At certain concentrations of $\mathrm{N}$, the anion sub-lattice can be represented as a row of anions, where $\mathrm{N}$ impurities substitute $\mathrm{P}$ atoms with the period equal to the Bohr diameter of the bound exciton in $\mathrm{GaP}$ (approximately $10 \mathrm{~nm}$ ) (Figure 5a). At some level of optical excitation, all the $\mathrm{N}$ sites will be filled by excitons, thereby creating an excitonic crystal (Figure 5b), which is a new phenomenon in solid-state physics and extremely interesting medium for application in optoelectronics and nonlinear optics [5-11].

Thus, using the bound excitons as short-lived analogues of atoms and sticking to some specific rules, including the necessity to build the excitonic superlattice with the identity

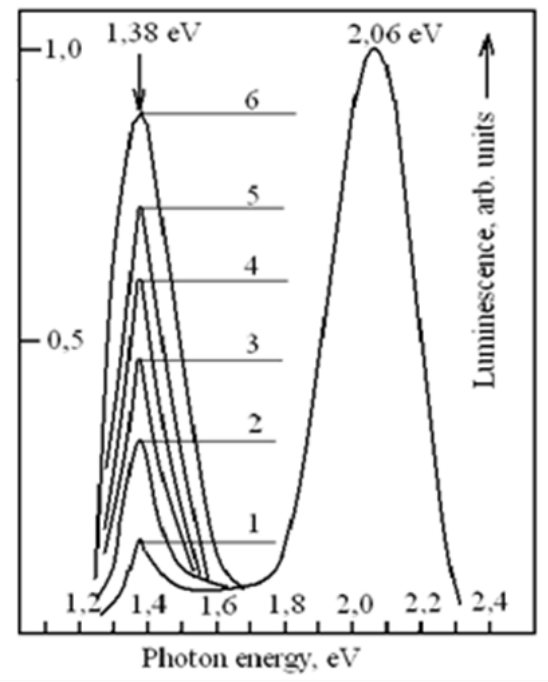

Figure 4: Luminescent spectra of GaP doped by Ge at $77.3 \mathrm{~K}$ and different excitation intensity Iex x 10-24 cm-2sec-1: 16(1); 10(2); 4,5(3); $2,3(4) ; 1,2(5)$; and $0,46(6)$.

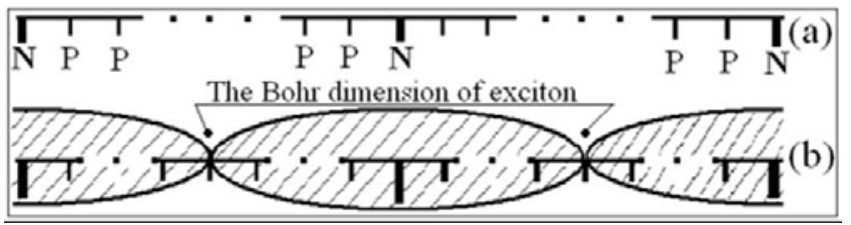

Figure 5: The models of 40 years ordered GaP doped by $\mathrm{N}$.

a. The new type of crystal lattice with periodic substitution of $\mathrm{N}$ atoms for the host $\mathrm{P}$ atoms.

b. The excitonic crystal on the base of this lattice. The substitution period is equal to the Bohr diameter of exciton $(\sim 100 \AA)$ and optical excitation is enough for complete saturation of the $\mathrm{N}$ sublattice (please see details in [6-11]). 
period equal to the bound exciton Bohr dimension in the GaP:N single crystal, we get a unique opportunity to create a new solid state medium consisting of short-lived nanoparticles - excitonic crystal with properties useful and interesting for application in optoelectronics, nanoscience and technology. According to the data extracted from our semicentennial study, inside this new medium the impurities in doped long-term ordered GaP create the sublattice with a period that depends on their concentration. Periodically substituting host atoms or occupying interstitial sites in the host lattice, the impurities become an intrinsic component of the modified crystal lattice and participate in the formation of new energy bands and phonon spectrum.

The ordered crystals with the host lattice modified by impurities could be very useful in various optoelectronic applications. Noted here are only a few potential applications in light emissive device structures. The properties of these structures will be very stable and time-independent. Uniform distribution of the recombination and activator centers at the optimum concentration yield maximum efficiency for light emission. Further investigations of the quasi-crystalline state of excitons (or bi-excitons) bound to an impurity superlattice with a period equal to the Bohr dimension will be very interesting and useful because they should strengthen greatly the nonlinear optical effects at low excitation intensities. This excitonic phase of high density of photons as an original accumulator of light also provides new opportunities for storage, transmission, and conversion of light.

With recent significant progress in semiconductor thin film deposition and growth techniques in some specific cases of optoelectronic device structure preparation, there will no longer be a need to wait for years for such ordering to occur. For instance, superlattice from GaP/GaP:N with the period of the order of the bound excitons (their Bohr radius is equal to 5 $\mathrm{nm}$ ) can be prepared by molecular beam epitaxy (MBE) or by MBE in combination with the laser-assisted epitaxy (LAE) [1214]. Furthermore, preparation of a two- or three-dimensional arrangement of $\mathrm{N}$ impurities in a GaP film is difficult but also possible with the help of ion lithography. Of course, this technique is at the frontier of current technological possibilities, at the same time, important progress in this sphere can be anticipated in the nearest future. In this case, we will get a unique opportunity to design 3-dimensional impurity superlattices with configuration, symmetry, and lattice that are optimal for application in a concrete device structure or for efficient realization of specific linear or nonlinear optical phenomena. In any case, independently of the method of creation, the impurity modified crystal lattices, and the excitonic (as well as bi-excitonic) phase with translational symmetry are very interesting objects, properties and possible application of which are now under our investigation.

Thus, the results of semi-centennial evolution of GaP:N properties are compiled here and in the references to this article. Novel and useful properties of GaP including expected similarity in behavior between nanoparticles and perfect bulk crystals, as well as very bright and broadband luminescence at room temperature, are observed. These results provide a new approach to the selection and preparation of perfect materials for optoelectronics [8] and a unique opportunity to realize a new form of solid-state host-the excitonic crystal [9-11]. Despite the fact that the time necessary for natural long-term ordering (years) is not optimal, the collected experience and results confirm expedience of the efforts directed to the formation in GaP of the $\mathrm{N}$ impurity superlattice having the identity period equal to the bound exciton dimension. As noted in Ref. [8], top quality material for industrial electronics can prepared by storing freshly grown crystals for years in a special storage (like aged wine, using the analogy familiar to Moldova's and other winemaking countries cultures). Only old crystals with the attained necessary properties will then be annually retrieved for device fabrication while new portions of fresh crystals will be placed for long-term ordering at room temperature.

Except natural aging of relevant crystals for years, preparation of the $\mathrm{N}$ superlattice for excitonic crystal can also be realized through the known methods of growth of multi-layer films, in particular, by molecular beam and laser-assisted epitaxy [12-14].

The excitonic crystal, created by the long-term ordering or by the noted above methods of growth of multi-layer films, as well as the bulk top quality GaP crystals with the unique optic properties, obtained by the long-term ordering process of freshly-prepared crystals, will be used in new generations of optoelectronic devices, sometimes instead of nanoparticles and a lot of other materials. In particular, keeping in mind the low energy of the bound exciton creation, one can expect a low threshold for the generation of non-linear optical effects in the excitonic crystal and a good opportunity to create new and very efficient optoelectronic devices.

Note that semiconductor nanoparticles were introduced into materials science and engineering mainly in order to avoid limitations inherent to freshly grown semiconductors with a lot of different defects. However, it was shown $[6,9]$ that this reason becomes unessential if, when justified, perfect longterm ordered semiconductor crystals are applied in electronics. Independently of their dimensions, they demonstrate very interesting for application properties. Therefore, tremendous commercial advantage can be achieved by using the long-term ordered, perfect GaP crystals or materials of similar behavior and properties instead of very expensive and labor-intensive technologies for diverse materials and their nanoparticles with spectral region and other parameters limited for application in the electronic industry (for details please see the papers [7-11]).

All the obtained results presented here and included in summary reviews [6-11] may sufficiently change the approach to the selection of materials necessary for electronics, and to make cheaper and simpler technology for the preparation of the selected materials and device structures based on them. This study of long-term convergence of bulk- and nanocrystal properties [6] brings a novel perspective to improving the quality of semiconductor crystals. My unique collection of pure and doped crystals of semiconductors grown in the 1960s provides an opportunity to observe long-term evolution of the properties 
of these key electronic materials.

During these more than half-centennial systematic investigations of our crystals, we have established the main trends in evolution of their optoelectronic and mechanical properties. It was shown that these stimuli to improve the quality of the crystal lattice are the consequence of thermodynamic driving forces and prevail over tendencies that would favor disorder and destruction. Our long-term ordered and therefore close to ideal crystals repeat the behavior of the best nanoparticles with pronounced quantum confinement effect.

For the first time, to the best of our knowledge, we have observed a new type of the crystal lattice where the host atoms occupy their proper (equilibrium) positions in the crystal field, while the impurities, once periodically inserted into the lattice, divide it in the short chains of equal length, where the host atoms perform harmonic vibrations. This periodic substitution of a host atom by an impurity allows the impurity to participate in the formation of the crystal's energy bands.

Besides the noted above commercial advantage of using in collaboration with competent specialists in the electronic industry the long-term ordered, perfect GaP crystals or materials of similar behavior and properties, we can jointly propose a novel approach to development of electronics and new generations of optoelectronic devices.

Of course, waiting for improvement of crystal quality for decades can be justified only in exceptional cases, thus we propose to turn it into an effective process for preparation of top quality material for industrial electronics, which due to its unique properties will be used in electronic devices substituting a multitude of imperfect freshly prepared materials.

\section{References}

1. Goryunova NA, Pyshkin SL, Borshchevskii AS, et al. Influence of Impurities and Crystallization Conditions on Growth of Platelet GaP Crystals. Symposium on Crystal Growth at the 7th Int Crystallography
Congress (Moscow, July 1966). J. Growth of Crystals. 1969;8:68-72.

2. Pyshkin SL. Preparation and Properties of Gallium Phosphide. Ph.D. thesis, State University of Moldova, Kishinev. 1967.

3. Pyshkin SL, Zifudin L. Excitons in highly optically excited gallium phosphide. J Luminescence1974; 9: 302-8.

4. Pyshkin SL (presented by Nobel Prize Laureate A.M. Prokhorov). Stimulated emission in gallium phosphide. J Soviet Physics-Doklady. 1975;19:845-846.

5. Pyshkin SL. Photoconductivity and luminescence of highly optically excited semiconductors. Dr. Sc. thesis, Lomonosov Moscow State University. 1978.

6. Pyshkin SL, Ballato J. Long-term convergence of bulk- and nanocrystal properties. In: Predeep P (ed.) Optoelectronics- Materials and Techniques2011. Rijeka: InTech; 2011. 459-76.

7. Pyshkin SL, Ballato J. Advanced light emissive device structures. In: Pyshkin SL and Ballato J (ed.) Optoelectronics-Advanced Materials and Devices. Rijeka: InTech; 2013. p. 1-24.

8. Pyshkin SL. Gallium Phosphide - New Prospect for Optoelectronics. Advances in Optoelectronic Materials. 2013; 1(4): 59-66.

9. Pyshkin S. Excitonic Crystal and Nanotechnology. Nanosci Technol. 2014;1(2):1-5.

10. Pyshkin SL. Excitonic Crystals and Perfect Semiconductors for Optoelectronics, In: Pyshkin SL and Ballato J (ed.) Optoelectronics Materials and Devices. Rijeka: InTech; 2015. p. 1-30.

11. Pyshkin SL. Excitonic Crystal, Nanotechnology and New Prospect for Optoelectronics. The Open Optics Journal. 2015; 9: 26-37.

12. Pyshkin SL, Fedoseev SA, Lagomarsino S, Giannini C. Preparation and structural properties of some III-V semiconductor films grown on (100) oriented Si substrates. J Appl Surf Sci. 1992;56-58:39-43.

13. Budyanu VA, Chechuy SN, Damaskin IA, Fedoseev SA, Nasakin AA, Pyshkin SL, et al. Investigation of $\mathrm{A}^{\mathrm{III}} \mathrm{B}^{\mathrm{v}}$-Si Heterojunctions Grown by Laser Deposition. Phys Stat Sol (a). 1985;91:737-44.

14. Budyanu VA, Chechuy SN, Pyshkin SL, et al. Laser vacuum epitaxy of III-V semiconductors on silicon. Rev Roumaine de Physique1987;32(1-2):216-9. 